\title{
Onlinekurse Autorenwerkstatt
}

\author{
J. Klewer ${ }^{1}$
}

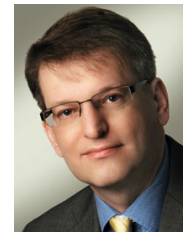

ten der Einstieg in wissenschaftliches Schreiben ermöglicht werden soll.

Daher finden Sie in dieser Ausgabe von HeilberufeSCIENCE drei wissenschaftliche Kurzmitteilungen und zwei Artikel. Zander widmet sich der Frage, warum Krankenpflegefachkräfte ihre Arbeitsplätze verlassen wollen und zeigt auf, welche Optionen dem Krankenhausmanagement zur Verfügung stehen, um dem Weggang von Pflegekräften entgegenzuwirken. Die Orientierungsschwierigkeiten von Patienten mit kognitiven Einschränkungen im Krankenhaus thematisieren Motzek et al. und weisen auf mögliche Strategien zur Vermeidung bzw. Kompensierung von Orientierungsschwierigkeiten hin. Die Betrachtung der Fremdeinschätzung von Schmerz bei Menschen mit dem Krankheitsbild Wachkoma durch Grubesic et al. lässt erkennen, dass sich die Einschätzung des Schmerzes von Bezugspflegekräften und weiteren Pflegekräften nicht unterscheidet. Voigt-Radloff et al. präsentieren die Ergebnisse einer Umfrage zu Optimierungspotenzialen in der regional vernetzten Versorgung und sensibilisieren für die Risiken einer fragmentierten Versorgung. Meyer et al. gehen auf die Gesundheit von Lehrkräften an Berufsschulen ein und zeigen Ansätze zur Optimierung der Gesundheitsförderung auf.

An dieser Stelle möchte ich Sie wie immer wieder einladen, die fünf Beiträge dieser Ausgabe von HeilberufeSCIENCE interessiert und kritisch zu lesen. Außerdem möchte ich Sie bitten, selbst wenn es sie nicht betreffen sollte, die Informationen zu den Onlinekursen der Autorenwerkstatt an potenzielle Autoren weiterzugeben.

SCIENCE, insbesondere über die Rubrik wissenschaftlic Kurzmitteilung, in welcher über kurze Beiträge zu Projek-

J. Klewer

heilberufescience@springer.com

1 Zwickau, Deutschland
Ihr

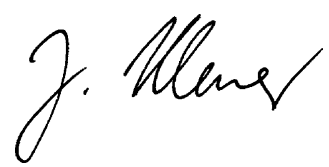

